Network Working Group

Request for Comments: 4578

Category: Informational
M. Johnston

Intel Corporation

S. Venaas, Ed.

UNINETT

November 2006

\title{
Dynamic Host Configuration Protocol (DHCP) Options for the Intel Preboot eXecution Environment (PXE)
}

Status of This Memo

This memo provides information for the Internet community. It does not specify an Internet standard of any kind. Distribution of this memo is unlimited.

Copyright Notice

Copyright (C) The IETF Trust (2006).

Abstract

We define Dynamic Host Configuration Protocol (DHCP) options being used by Preboot execution Environment (PXE) and Extensible Firmware Interface (EFI) clients to uniquely identify booting client machines and their pre-OS runtime environment so that the DHCP and/or PXE boot server can return the correct os bootstrap image (or pre-boot application) name and server to the client.

Table of Contents

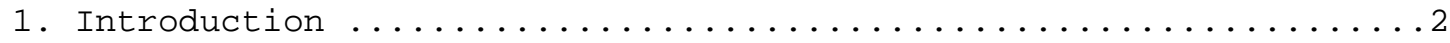

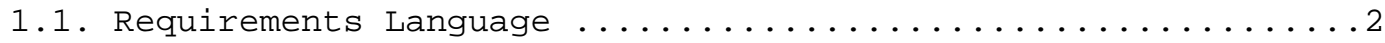

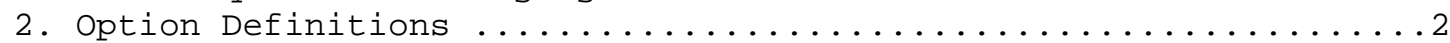

2.1. Client System Architecture Type Option Definition ........2

2.2. Client Network Interface Identifier Option Definition .....3

2.3. Client Machine Identifier Option Definition ..........4

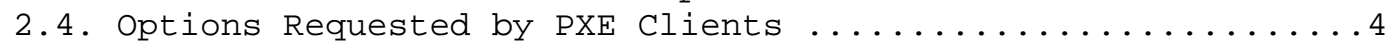

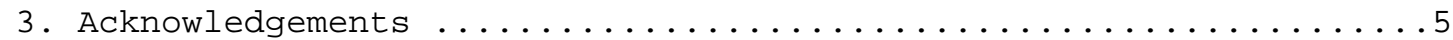

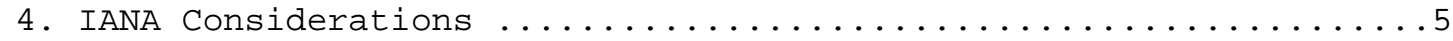

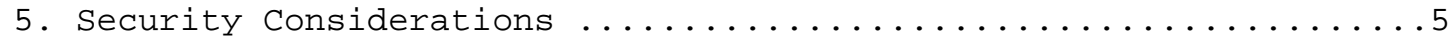

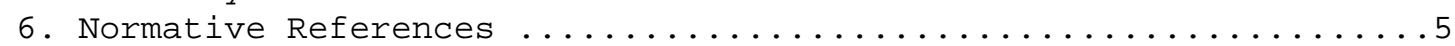




\section{Introduction}

These DHCP [2] options are being widely used by PXE-compliant clients to uniquely identify booting client machines themselves and their pre-OS runtime environment so that the DHCP and/or PXE boot server can return the correct os bootstrap image (or pre-boot application) name and server to the client. In the past, this work was done by examining the network Media Access Code (MAC) address in the "chaddr" field in the BOOTP/ DHCP header and keeping a database of MAC addresses on the BOOTP/DHCP server. This was deemed insufficient for large and complex networks for two main reasons. 1) Multiple laptops could end up with the same MAC address if the network interface was in a shared docking station. 2) Multiple network devices and MAC addresses could be used by one machine for redundancy or because of repairs. Another issue that came up was the machine that could change its pre-os runtime environment. This issue caused the creation of another new option to identify the runtime environment so that the correct binary image could be matched up with the booting machine. These options are defined by Intel in the PXE [3] and EFI [4] specifications and are being documented in this draft for completeness within the IETF.

\subsection{Requirements Language}

The key words "MUST", "MUST NOT", "REQUIRED", "SHALL", "SHALL NOT", "SHOULD", "SHOULD NOT", "RECOMMENDED", "MAY", and "OPTIONAL" in this document are to be interpreted as described in RFC 2119 [1] .

2. Option Definitions

There are three DHCP options [5] defined for use by PXE clients.

\subsection{Client System Architecture Type Option Definition}

The format of the option is:

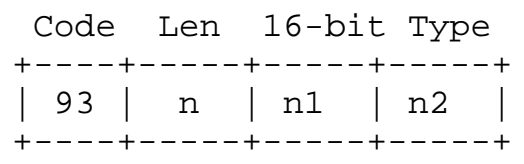


Octet "n" gives the number of octets containing "architecture types" (not including the code and len fields). It MUST be an even number greater than zero. Clients that support more than one architecture type MAY include a list of these types in their initial DHCP and PXE boot server packets. The list of supported architecture types MAY be reduced in any packet exchange between the client and server (s). octets "n1" and "n2" encode a 16-bit architecture type identifier that describes the pre-boot runtime environment(s) of the client machine.

As of the writing of this document, the following pre-boot architecture types have been requested.

$\begin{array}{cl}\text { Type } & \text { Architecture Name } \\ --- & ------------ \\ 0 & \text { Intel x86PC } \\ 1 & \text { NEC/PC98 } \\ 2 & \text { EFI Itanium } \\ 3 & \text { DEC Alpha } \\ 4 & \text { Arc x86 } \\ 5 & \text { Intel Lean Client } \\ 6 & \text { EFI IA32 } \\ 7 & \text { EFI BC } \\ 8 & \text { EFI XsCale } \\ 9 & \text { EFI x86-64 }\end{array}$

This option MUST be present in all DHCP and PXE packets sent by PXEcompliant clients and servers.

\subsection{Client Network Interface Identifier Option Definition}

The format of the option is:

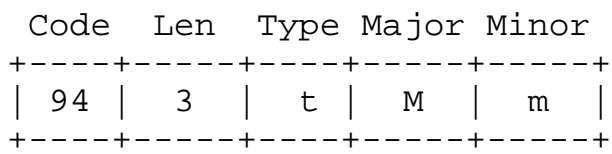

Octet "t" encodes a network interface type. For now the only supported value is 1 for Universal Network Device Interface (UNDI). octets "M" and "m" describe the interface revision. To encode the UNDI revision of 2.11, "M" would be set to 2, and "m" would be set to 11 (OxOB). 


\begin{tabular}{|c|c|}
\hline Revision & Description \\
\hline$<2.00$ & LANDesk service agent boot ROMs. \\
\hline 2.00 & First generation PXE boot ROMs. \\
\hline 2.01 & Second generation PXE boot ROMs. \\
\hline 3.00 & $\begin{array}{l}\text { 32/64-bit UNDI specification. } \\
\text { EFI boot services driver only. } \\
\text { No EFI runtime support. }\end{array}$ \\
\hline 3.10 & $\begin{array}{l}\text { 32/64-bit UNDI specification. (Beta) [4] } \\
\text { First generation EFI runtime driver support. }\end{array}$ \\
\hline 3.20 & $\begin{array}{l}\text { 32/64-bit UNDI specification. (Release) [4] } \\
\text { Second generation EFI runtime driver support. }\end{array}$ \\
\hline
\end{tabular}

This option MUST be present in all DHCP and PXE packets sent by PXEcompliant clients and servers.

\subsection{Client Machine Identifier Option Definition}

The format of the option is:

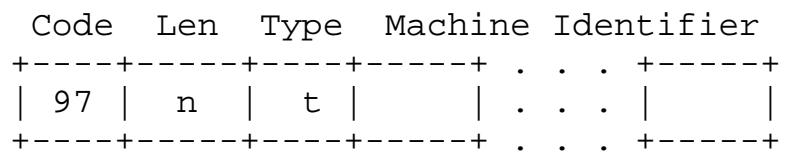

Octet "t" describes the type of the machine identifier in the remaining octets in this option. 0 (zero) is the only value defined for this octet at the present time, and it describes the remaining octets as a 16-octet Globally Unique Identifier (GUID). Octet "n" is 17 for type 0. (One definition of GUID can be found in Appendix A of the EFI specification [4].)

This option MUST be present in all DHCP and PXE packets sent by PXEcompliant clients and servers.

\subsection{Options Requested by PXE Clients}

All compliant PXE clients MUST include a request for DHCP options 128 through 135 in all DHCP and PXE packets. The format and contents of these options are NOT defined by the PXE specification. These options MAY be present in the DHCP and PXE boot server replies and are meant for use by the downloaded network bootstrap programs. These options are NOT used by the PXE boot ROMs. 
As options 128-135 are not officially assigned for PXE use (before November 2004 they were considered site-specific options, [6]), use of these option values for PXE may conflict with other uses of the same options on the same networks.

3. Acknowledgements

The authors thank Bernie Volz for valuable input.

4. IANA Considerations

IANA has updated the numbering space defined for public DHCP options in [7] with references to this document for options 93, 94, and 97 (previously, there were references to [8]). Also, IANA marked options 128-135 as being used by PXE and referenced this document.

5. Security Considerations

By specifying incorrect values for some of these options, a client may get access to, and possibly attempt to execute, code intended for another platform or client. This may have security ramifications. Also note that these options contain information about a client's system architecture and pre-os runtime environment that is revealed to anyone who is able to listen in on DHCP messages sent by the client. This information may be of use to potential attackers.

6. Normative References

[1] Bradner, S., "Key words for use in RFCs to Indicate Requirement Levels", BCP 14, RFC 2119, March 1997.

[2] Droms, R., "Dynamic Host Configuration Protocol", RFC 2131, March 1997.

[3] Henry, M. and M. Johnston, "Preboot Execution Environment (PXE) Specification", September 1999, <http://www.pix.net/software/pxeboot/archive/pxespec.pdf>.

[4] Intel Corp., "Extensible Firmware Interface Specification", December 2002, <http://developer.intel.com/technology/efi/ main_specification.htm>.

[5] Alexander, S. and R. Droms, "DHCP Options and BOOTP Vendor Extensions", RFC 2132, March 1997.

[6] Volz, B., "Reclassifying Dynamic Host Configuration Protocol version 4 (DHCPv4) Options", RFC 3942, November 2004. 
[7] Droms, R., "Procedures and IANA Guidelines for Definition of New DHCP Options and Message Types", BCP 43, RFC 2939, september 2000 .

[8] Droms, R., "Unused Dynamic Host Configuration Protocol (DHCP) Option Codes", RFC 3679, January 2004.

Authors' Addresses

Michael Johnston

Intel Corporation

MS. JF1-239 $2111 \mathrm{NE}$ 25th Ave.

Hillsboro, OR 97124

USA

Phone: +1 503-264-9703

EMail: michael.johnstondintel.com

Stig Venaas

UNINETT

Trondheim NO-7465

Norway

EMail: venaas@uninett.no 
Full Copyright statement

Copyright (C) The IETF Trust (2006).

This document is subject to the rights, licenses and restrictions contained in BCP 78, and except as set forth therein, the authors retain all their rights.

This document and the information contained herein are provided on an "AS IS" basis and THE CONTRIBUTOR, THE ORGANIZATION HE/SHE REPRESENTS OR IS SPONSORED BY (IF ANY), THE INTERNET SOCIETY, THE IETF TRUST, AND THE INTERNET ENGINEERING TASK FORCE DISCLAIM ALL WARRANTIES, EXPRESS OR IMPLIED, INCLUDING BUT NOT LIMITED TO ANY WARRANTY THAT THE USE OF THE INFORMATION HEREIN WILL NOT INFRINGE ANY RIGHTS OR ANY IMPLIED WARRANTIES OF MERCHANTABILITY OR FITNESS FOR A PARTICULAR PURPOSE.

Intellectual Property

The IETF takes no position regarding the validity or scope of any Intellectual Property Rights or other rights that might be claimed to pertain to the implementation or use of the technology described in this document or the extent to which any license under such rights might or might not be available; nor does it represent that it has made any independent effort to identify any such rights. Information on the procedures with respect to rights in RFC documents can be found in BCP 78 and BCP 79 .

Copies of IPR disclosures made to the IETF Secretariat and any assurances of licenses to be made available, or the result of an attempt made to obtain a general license or permission for the use of such proprietary rights by implementers or users of this specification can be obtained from the IETF on-line IPR repository at http://www.ietf.org/ipr.

The IETF invites any interested party to bring to its attention any copyrights, patents or patent applications, or other proprietary rights that may cover technology that may be required to implement this standard. Please address the information to the IETF at ietf-ipreietf.org.

Acknowledgement

Funding for the RFC Editor function is currently provided by the Internet Society. 